\title{
Biochemical and ultrastructural changes in rabbit sclera after treatment with 7-methylxanthine, theobromine, acetazolamide, or L-ornithine
}

\author{
Klaus Trier, Elith Bjarne Olsen, Takasi Kobayashi, Søren Munk Ribel-Madsen
}

\begin{abstract}
Aims-To examine a possible effect of 7-methylxanthine, theobromine, acetazolamide, or L-ornithine on the ultrastructure and biochemical composition of rabbit sclera.

Methods-Groups of pigmented rabbits,
\end{abstract} six in each group, were dosed during 10 weeks with one of the substances under investigation, and one untreated group was the control. Samples of anterior and posterior sclera were taken for determination of hydroxyproline, hydroxylysine, proline, proteoglycans, uronic acids and dermatan sulphate, chondroitin sulphate, and hyaluronic acid. Sections were examined with electron microscopy, and the diameter of the individual collagen fibrils was measured.

Results-Treatment with theobromine produced a significant increase in the contents of hydroxylysine, hydroxyproline, and proline in both anterior and posterior sclera, while 7-methylxanthine increased the contents of hydroxyproline and proline selectively in posterior sclera. Acetazolamide, on the other hand, significantly decreased the contents of hydroxyproline and proline in samples from anterior sclera. Uronic acids in both anterior and posterior sclera were significantly reduced by treatment with 7-methylxanthine, and L-ornithine significantly reduced uronic acids in posterior sclera. An inverse correlation between contents of hydroxyproline and uronic acids was found. The mean diameter of collagen fibrils was significantly higher in the posterior sclera from rabbits treated with 7-methylxanthine or theobromine, and significantly lower in rabbits treated with acetazolamide or L-ornithine compared with controls. In the anterior sclera, fibril diameter was significantly reduced in all treatment groups compared with controls. A positive, significant correlation between fibril diameter and content of hydroxyproline and proline was found in posterior sclera.

Conclusion-7-Methylxanthine, a metabolite of caffeine, increases collagen concentration and the diameter of collagen fibrils in the posterior sclera, and may be useful for treatment or prevention of conditions associated with low level and/or inferior quality of scleral collagen, such as axial myopia, chronic open angle glau- coma, and possibly neovascular age related macular degeneration. The apparent loss of collagen induced by chronic treatment with acetazolamide should be taken into consideration as a potentially harmful side effect. These results may indicate that scleral biochemistry and ultrastructure are influenced by the retinal pigment epithelium. One possible explanation is that the scleral fibroblasts which produce the collagen are sensitive to changes in the physiological electric field created by the retinal pigment epithelium.

(Br f Ophthalmol 1999;83:1370-1375)

Sclera supports the visual apparatus, and disturbances in its biomechanical properties may lead to excessive elongation of the eye (axial myopia). The ciliary arteries and vortex veins traverse the sclera and may be vulnerable to the increased rigidity of this tissue in conditions associated with disturbed choroidal blood flow, such as glaucoma and the neovascular form of age related macular degeneration.

Earlier research by this group indicates that long term treatment with caffeine $(1,3,7-$ trimethylxanthine) induces significant changes in the biochemistry and ultrastructure of rabbit sclera (unpublished).

In the present study, we investigated whether long term treatment with theobromine $(3,7-$ dimethylxanthine) and 7-methylxanthine, both metabolites of caffeine, have a similar effect.

Among other effects, caffeine affects the function of the retinal pigment epithelium, as judged by its effect on electrophysiological variables such as electro-oculography (standing potential) or electroretinography (ERG). ${ }^{1}$

Attempts to determine the effect of 7-methylxanthine and theobromine on the electrophysiology of the eye in an animal model were unsuccessful, because the substances are insoluble at neutral $\mathrm{pH}$, but it could be confirmed that caffeine increases the standing potential and c-wave of ERG (Sven Erik Nilsson, University of Linköping, Sweden, personal communication).

Two substances that are known to reduce the standing potential-namely, acetazolamide and L-ornithine, were included in the study. Acetazolamide is well known to reduce the standing potential and c-wave of ERG generated by the retinal pigment epithelium. ${ }^{2}{ }^{3}$

L-ornithine has been reported to reduce the standing potential and c-wave of ERG. ${ }^{4}$ 
Materials and methods

ANIMALS AND TREATMENT

Female, pigmented rabbits from the strain denoted "Chinchilla bastard, Charles River Deutschland" were used. Their age at the beginning of the experiment was 8 weeks, and their body weight $1.4-1.9 \mathrm{~kg}$. The rabbits were kept in a university experimental animal facility under veterinary supervision. The light/ dark cycles were 12/12 hours.

The animals were fed a standard rabbit diet (Stanrab, Special Diet Services, Witham, Essex) in an amount of $75 \mathrm{~g}$ per day at the start of the study, increasing to $130 \mathrm{~g}$ per day during the last week. The rabbits had been divided randomly into treatment groups of six animals, with no significant differences between their mean body weights. One group served as control and four groups were given one of the substances under investigation. These substances had been homogeneously added to the feed by the manufacturer in the following amounts: 7-methylxanthine or theobromine (Sigma, St Louis, MO, USA) or acetazolamide (crushed Diamox tablets, Wyeth Lederle, Philadelphia, PA, USA), in an amount of $500 \mathrm{mg}$ active substance per $\mathrm{kg}$ standard diet, resulting in a daily dose of $38 \mathrm{mg}$ increasing to $65 \mathrm{mg}$, corresponding to approximately $30 \mathrm{mg}$ per $\mathrm{kg}$ body weight per day throughout the 10 week study period; L-ornithine (Sigma) in an amount of $4.5 \mathrm{~g}$ per kg standard diet, resulting in a daily dose of $340 \mathrm{mg}$ increasing to $585 \mathrm{mg}$ in addition to normal dietary intake, corresponding to approximately $240 \mathrm{mg}$ per $\mathrm{kg}$ increasing to $310 \mathrm{mg}$ per $\mathrm{kg}$ body weight.

SAMPLES

The rabbits were killed after 10 weeks by intravenous pentobarbitone. The right eye from each animal was dissected out and samples taken from the same quadrant. A $5 \times 10 \mathrm{~mm}$ biopsy from the anterior sclera was cut along the limbus, without the cornea, and a punch biopsy $8 \mathrm{~mm}$ in diameter was taken from the posterior sclera. Each sample was divided in three pieces, for determination of collagen related amino acids, proteoglycans, and uronic acids and glycosaminoglycans, respectively, and the pieces were immediately put in acetone and stored cold until processing and analysis. Samples with a diameter of $4 \mathrm{~mm}$ were taken from the anterior and posterior part of sclera from one randomly chosen animal in each experimental group for electron microscopy.

COLLAGEN

The pieces of sclera were delipidised in four changes of acetone and diethyl ether and dried under vacuum to constant weight. Each dry biopsy sample was cut into chips approximately $0.5 \mathrm{~mm}$ thick and divided equally in two portions for duplicate processing. The aliquots were weighed separately and then taken to vapour phase, acid hydrolysis in an oxygen free atmosphere at $114^{\circ} \mathrm{C}$ for 24 hours. The resulting hydrolysates were evaporated to dryness and redissolved in $0.1 \mathrm{M}$ hydrochloric acid by standing overnight at $4^{\circ} \mathrm{C}$, after which three aliquots each representing 1/40 of the total volume of the hydrolysate and three aliquots representing $1 / 8$ were mixed with a solution of citrulline, used as internal standard, evaporated to dryness, and reacted with phenylisothiocyanate. The resulting derivatives were analysed in an HPLC (high performance liquid chromatography) system using Hypersil BDS C18 $3 \mu \mathrm{m} 150 \times 4.6 \mathrm{~mm}$ column (Shandon, Runcorn, Cheshire) eluted with a gradually increasing concentration from acetonitrile in aqueous sodium acetate buffer $0.1 \mathrm{~mol} / 1$ with triethylamine $4 \mathrm{~m} / \mathrm{mol}, \mathrm{pH} 5.70$. The ultraviolet absorbance of the eluate from the column was monitored at a wavelength of 254 $\mathrm{nm}$. The calibration of the HPLC system for result processing was based on the results referring to equal analysis and calculation of standard samples, four samples at each of 10 levels within the relevant range $0.5-130 \mathrm{nmol}$ from each of 19 amino acids per sample. The standard samples were made from the "Amino Acid Standard H" (Pierce, Rockford, IL, USA), L-4-hydroxyproline (Merck, Darmstadt, Germany) and 5-hydroxylysine (Aldrich, Steinheim, Germany). Amounts of hydroxyproline and proline were calculated as the mean of the determinations of triplicate aliquots of $1 / 40$ of the redissolved hydrolysate, hydroxylysine was quantitated from the analysis of $1 / 8$ aliquots, and all contents were then calculated per mg dry, delipidised tissue.

\section{PROTEOGLYCANS}

Proteoglycan content was determined with a dot blot adaption of the alcian blue method of Björnsson. ${ }^{5}$

The tissue samples were extracted with $20 \mu \mathrm{l}$ $4 \mathrm{M}$ guanidine- $\mathrm{HCl}, 50 \mathrm{mM}$ sodium acetate $\mathrm{pH} 4.8$ per mg wet weight overnight at room temperature. Extracted samples were centrifuged to remove debris. Twenty $\mu$ l of supernatant were diluted $1 / 10$ in extraction buffer and $20 \mu \mathrm{l}$ of diluted supernatant mixed with $10 \mu \mathrm{l}$ reagent $1^{5}$ on a shaking table for 15 minutes. Two hundred $\mu$ l of reagent $2^{5}$ were added, and samples were incubated for 60 minutes to precipitate the proteoglycans. The proteoglycan/ alcian blue precipitate was transferred to a 96 well MillBlotD apparatus and washed with $2 \times$ $200 \mu \mathrm{l} 40 \%$ DMSO, $0.05 \mathrm{M} \mathrm{MgCl}_{2}$. Colour intensity in dot blots was quantified by the use of a Microtek E3 scanner and Scan Analysis software (Biosoft, Cambridge). Chondroitin-6sulphate (Sigma C4384) was used as calibrator. All samples were analysed in duplicate. The results were expressed as $\mu$ g proteoglycan per mg tissue (wet weight). Analysis for proteoglycans was performed by Wieslab AB, Ideon, S-223 70 Lund, Sweden.

\section{URONIC ACIDS}

Uronic acid analysis was performed by the procedure described by Blumencrantz and Asboe-Hansen using glucuronic acid as standard. ${ }^{6}$ The quantitative determination gives a quantitative measure of glycosaminoglycans present since glucuronic acid and iduronic acid are constituent parts of these. 
GLYCOSAMINOGLYCANS

The glycosaminoglycans were separated by cellulose acetate electrophoresis as reported previously. ${ }^{7}$ The samples were digested with proteolytic enzymes (Pronase E, Merck, Darmstadt, Germany). A raw mixture of glycosaminoglycans was then precipitated by ethanol and redissolved in two portions of $0.05 \mathrm{M}$ sodium acetate buffer $\mathrm{pH} 5.00$, and glycosaminoglycans were precipitated from the combined supernatants by addition of N-cetyl$\mathrm{N}, \mathrm{N}, \mathrm{N}$-trimethyl-ammonium bromide and standing for 16 hours. Remnants from the precipitation agent were removed by three ethanol washings.

Before electrophoresis the purified isolates were dissolved in $0.01 \mathrm{M}$ sodium hydroxide solution. Cellulose acetate plates $60 \times 76 \mathrm{~mm}$ (Titan III, Helena Laboratories, UK) were prepared for use by immersing $20 \mathrm{~mm}$ of one end in water and the opposite end in a $0.02 \mathrm{M}$ barium acetate buffer solution $\mathrm{pH}$ 4.00, leaving a $2-3 \mathrm{~mm}$ wide band apparently dry between water and buffer zone. The solutions from unknown glycosaminoglycan samples and a range of concentrations of a standard mixture of dermatan sulphate, chondroitin sulphate $\mathrm{C}$, heparan sulphate, and hyaluronic acid (all from Sigma) were applied on the plates as $2 \mu$ spots.

Electrophoresis was performed at $8^{\circ} \mathrm{C}$, with $250 \mathrm{~V}$ constant voltage. The buffer tanks were filled with $0.2 \mathrm{M}$ barium acetate buffer solution $\mathrm{pH}$ 4.00. The electrical field across the plates was switched on for periods of 4 minutes, 10 minutes, 15 minutes, and finally 45 minutes, interrupted by immersions of the plates into $0.2 \mathrm{M}$ barium acetate buffer solution $\mathrm{pH} 4.00$ with increasing concentrations of ethanol, in order to utilise a different end points of individual types of glycosaminoglycans with ethanol. The plates were then stained in a $0.1 \%$ solution of alcian blue 8GS (Chroma-Gesellschaft Schmid, Köngen, Germany) and destained.

The plates were scanned while still humid in light transmission mode, under standardised conditions of magnification, brightness, and contrast. The images were analysed with the SigmaGel program (Jandel Scientific Software, SPSS Scientific Products, Chicago, IL, USA). The area under the peaks from dermatan sulphate, chondroitin sulphate, and hyaluronic acid in the intensity profile were calculated. Results from the standards on every plate were used for construction of a calibration curve for each glycosaminoglycan. The identity of the mentioned glycosaminoglycans in representative isolates from sclera was verified by sequential hydrolysis with chondroitinase AC and chondroitinase B (Seikagaku, Tokyo, Japan) and hyaluronidase (Boehringer Mannheim, Germany).

ELECTRON MICROSCOPY AND SCLERAL THICKNESS The examiner was informed that the samples were from sclera, but was not aware of the nature of the study nor of the identity of the individual samples.
To obtain an exact surface for the perpendicular sectioning, the epoxy embedded blocks of sclera were easily oriented under a binocular microscope built in an ultramicrotome. Semithin epon sections, $2 \mu \mathrm{m}$ thick, were cut and stained by toluidine blue and studied by a light microscope.

For ultramicrotomy, the pyramids were cut in the middle of the sclera under the light microscopy from the semithin sections.

For ultrastructural studies, the specimens were fixed in $4 \%$ glutaraldehyde solution of caccodylate buffer $\mathrm{pH} 7.4$ with $7.5 \%$ sucrose at $4^{\circ} \mathrm{C}$ overnight, osmicated in a $1 \%$ osmic acid solution of phosphate buffered saline $\mathrm{pH}$ 7.4. The specimens were then dehydrated in a series of increased concentration of ethanol and embedded in epoxy resin.

Semithin sections were stained by $0.5 \%$ toluidine blue and studied by light microscopy. No inflammatory changes were found in the specimens.

The thickness of sclera was measured from basal the lamina of the choroid to the outer surface at five places with $0.1 \mathrm{~mm}$ wide intervals for each sample.

Ultrathin sections were cut from the middle part of sclera and stained by uranyl acetate and lead citrate for routine electron microscopy. An electron microscope Jeol 100 CX was operated at $80 \mathrm{kV}$ for observation.

For morphometric analysis of collagen fibrils, electron micrographs, taken in randomly selected areas of the middle layer of sclera at a direct magnification of 20000 and enlarged three times photographically, were used. The number of collagen fibrils of various thicknesses were counted in randomly selected areas of $19 \mu \mathrm{m}^{2}$ for each experimental group.

\section{STATISTICS}

The results were analysed by the sPSS (SPSS Inc, Chicago, IL, USA).

Mean values from the four treatment groups and the control group were compared by one way analysis of variance with the Bonferroni post hoc multiple comparisons test, and by the Kruskal-Wallis $\mathrm{H}$ test in the cases where Levene's test had revealed that variances were not homogeneous.

Comparisons in pairs of treatment groups were done by Student's $t$ test or, in cases of inhomogeneity of variance, by exact MannWhitney test.

Normality of distribution of variable values in samples from each location was tested with Kolmogorov-Smirnov (Lilliefors) test, and after normality was not rejected the Pearson correlation between such variables was calculated.

\section{Results}

GENEREL DEVELOPMENT OF ANIMALS

The gain in body weight in the groups were: control, mean 1.02 (SD 0.24) kg; 7-methylxanthine, mean $1.16(0.12) \mathrm{kg}$; theobromine, $1.12(0.09) \mathrm{kg}$; acetazolamide, 1.00 (0.11) kg; L-ornithine, $1.05(0.17) \mathrm{kg}$. No significant differences were present between these groups. 
Table 1 Scleral thickness $(\mathrm{mm})$ in sample taken for electron microscopy

\begin{tabular}{lll}
\hline & $\begin{array}{l}\text { Anterior sclera } \\
\text { Mean }(S D)\end{array}$ & $\begin{array}{l}\text { Posterior sclera } \\
\text { Mean }(S D)\end{array}$ \\
\hline Control & $0.29(0.019)$ & $0.33(0.018)$ \\
7-Methylxanthine & $0.32(0.024)$ & $0.37(0.042)$ \\
Theobromine & $0.47(0.050) \mathrm{p}<0.001^{\star}$ & $0.32(0.026)$ \\
Acetazolamide & $0.28(0.030)$ & $0.27(0.010) \mathrm{p}=0.009^{\star}$ \\
L-ornithine & $0.39(0.076) \mathrm{p}=0.015^{\star}$ & $0.34(0.018)$ \\
\hline
\end{tabular}

${ }^{\star} \mathrm{p}$ Values are significance of difference from control in ANOVA with Bonferroni test.

The weight of the total, right eye immediately after it was dissected out was: control, mean 2.98 (0.18) g; 7-methylxanthine, mean $3.08(0.12) \mathrm{g}$; theobromine, $2.95(0.08) \mathrm{g}$; acetazolamide, $3.02(0.15) \mathrm{g}$; L-ornithine, 2.97 (0.14) g. No significant differences were present between the groups as to the weight of the eye.

\section{SCLERAL THICKNESS}

The thickness of the samples taken for electron microscopy are shown in Table 1 . The anterior sclera was significantly thicker in animals treated with theobromine and L-ornithine compared with control. The posterior sclera was significantly thinner in animals treated with acetazolamide compared with control.

COLLAGEN RELATED AMINO ACIDS

The contents determined are given in Table 2 for the anterior and posterior part of the sclera. A significant difference between the five groups was demonstrated as to all three amino acids in both locations, with the $\mathrm{p}$ values stated. Comparisons of each treatment group with controls revealed that animals treated with theobromine had significantly higher contents of hydroxyproline and proline in the anterior sclera and significantly higher contents of hydroxyproline, hydroxylysine, and proline in the posterior sclera. Animals treated with 7-methylxanthine showed significantly higher contents of hydroxyproline and proline in posterior sclera compared with controls, while no significant change was found in the anterior sclera. Treatment with acetazolamide significantly reduced the contents of hydroxyproline and proline in anterior sclera compared with control.

PROTEOGLYCANS

No significant differences were found regarding the contents of proteoglycans in the sclera samples (Table 3), but there was a near signifi-

Table 2 Content of hydroxyproline, hydroxylysine, and proline (nmol/mg tissue, dried and defatted weight) from anterior and posterior sclera

\begin{tabular}{llll}
\hline & $\begin{array}{l}\text { Hydroxyproline } \\
\text { Mean (SD) }\end{array}$ & $\begin{array}{l}\text { Hydroxylysine } \\
\text { Mean (SD) }\end{array}$ & $\begin{array}{l}\text { Proline } \\
\text { Mean (SD) }\end{array}$ \\
\hline $\begin{array}{l}\text { Anterior part of sclera: } \\
\text { Control }\end{array}$ & $638(24)$ & $45(10)$ & $928(56)$ \\
7-Methylxanthine & $667(73)$ & $45(3)$ & $951(111)$ \\
Theobromine & $751(53) \mathrm{p}=0.004^{\star}$ & $49(6)$ & $1073(78) \mathrm{p}=0.004^{\star}$ \\
Acetazolamide & $596(27) \mathrm{p}=0.016^{\star}$ & $41(4)$ & $843(47) \mathrm{p}=0.018^{\star}$ \\
L-ornithine & $605(37)$ & $37(3)$ & $875(48)$ \\
p Value in Kruskal-Wallis H test & 0.0024 & 0.0150 & 0.0030 \\
$\begin{array}{l}\text { Posterior part of sclera: } \\
\text { Control }\end{array}$ & $621(59)$ & $41(5)$ & $864(87)$ \\
7-Methylxanthine & $719(81) \mathrm{p}=0.039^{\star}$ & $44(7)$ & $1018(108) \mathrm{p}=0.022^{\star}$ \\
Theobromine & $741(18) \mathrm{p}=0.001^{\star}$ & $49(4) \mathrm{p}=0.01^{\star}$ & $1029(45) \mathrm{p}=0.002^{\star}$ \\
Acetazolamide & $563(91)$ & $38(6)$ & $781(125)$ \\
L-ornithine & $611(24)$ & $38(5)$ & $871(35)$ \\
p Value in Kruskal-Wallis H test & 0.0009 & 0.0150 & 0.0010 \\
\hline
\end{tabular}

^Indicates $\mathrm{p}$ values from Student's $t$ test are stated where $<0.05$ in comparison with control.
Table 3 Content of proteoglycans ( $\mu \mathrm{g} / \mathrm{mg}$ tissue, wet weight)

\begin{tabular}{lll}
\hline & $\begin{array}{l}\text { Anterior sclera } \\
\text { Mean }(S D)\end{array}$ & $\begin{array}{l}\text { Posterior sclera } \\
\text { Mean }(S D)\end{array}$ \\
\hline Control & $2.9(0.5)$ & $2.9(0.4)$ \\
7-Methylxanthine & $2.5(0.5)$ & $3.3(0.3)$ \\
Theobromine & $2.6(0.7)$ & $2.5(0.4)$ \\
Acetazolamide & $2.9(0.4)$ & $2.8(0.8)$ \\
L-ornithine & $3.0(1.1)$ & $3.0(0.9)$
\end{tabular}

No significant differences in comparisons with control.

Table 4 Content of uronic acid ( $\mu$ g/mg tissue, dry weight)

\begin{tabular}{lll}
\hline & $\begin{array}{l}\text { Anterior sclera } \\
\text { Mean }(S D)\end{array}$ & $\begin{array}{l}\text { Posterior sclera } \\
\text { Mean }(S D)\end{array}$ \\
\hline Control & $2.28(0.22)$ & $2.21(0.33)$ \\
7-Methylxanthine & $1.85(0.33) \mathrm{p}=0.024^{\star}$ & $1.63(0.23) \mathrm{p}=0.005^{\star}$ \\
Theobromine & $2.16(0.28)$ & $1.86(0.52)$ \\
Acetazolamide & $2.17(0.29)$ & $1.94(0.19)$ \\
L-ornithine & $2.13(0.36)$ & $1.73(0.38) \mathrm{p}=0.037^{\star}$ \\
\hline
\end{tabular}

${ }^{\star} \mathrm{p}<0.05$.

cant decrease in the posterior sclera from the animals which had received theobromine, $\mathrm{p}=0.05$ in exact Mann-Whitney test.

URONIC ACIDS

The results with uronic acids are given in Table 4 . The contents in both the anterior and posterior sclera were significantly reduced by treatment with 7-methylxanthine, and contents in the posterior sclera were significantly reduced in the group treated with L-ornithine.

The correlation between the contents of uronic acids and hydroxyproline or proline was examined, and an inverse correlation was seen between uronic acids and hydroxyproline; however, it did not reach significance.

\section{GLYCOSAMINOGLYCANS}

Dermatan sulphate, chondroitin sulphate, and small amounts of hyaluronic acid were found in the scleral samples. The analysis of variance with multiple comparisons test, referring to each of the three components in the anterior or posterior part, showed no overall difference between the four treatment groups and the controls. The highest values for dermatan sulphate were found in the anterior and posterior sclera of animals treated with 7-methylxanthine, and the differences with controls nearly reached significant levels, $\mathrm{p}=0.053$ and 0.055 , respectively (Table 5).

\section{ELECTRON MICROSCOPY}

The mean diameter of collagen fibrils in the anterior sclera was significantly reduced in all treatment groups compared with controls $(\mathrm{p}<0.0001)$. In the posterior sclera, the mean diameter of collagen fibrils was significantly larger in rabbits treated with 7-methylxanthine

Table 5 Content of dermatan sulphate (ng/mg tissue, dry weight)

\begin{tabular}{|c|c|c|}
\hline & $\begin{array}{l}\text { Anterior sclera } \\
\text { Mean (SD) }\end{array}$ & $\begin{array}{l}\text { Posterior sclera } \\
\text { Mean (SD) }\end{array}$ \\
\hline Control & $1988(623)$ & $1910(361)$ \\
\hline 7-Methylxanthine & $2676(451) \mathrm{p}=0.053$ & $2766(1060) \mathrm{p}=0.055$ \\
\hline Theobromine & $2365(657)$ & $2216(687)$ \\
\hline Acetazolamide & $2231(575)$ & $1920(499)$ \\
\hline L-ornithine & $2133(487)$ & $1967(481)$ \\
\hline
\end{tabular}

$\mathrm{p}$ Values from Student's $t$ test or exact Mann-Whitney test. 
Table 6 Mean diameter of collagen fibrils ( $\mathrm{nm}$ )

\begin{tabular}{lll}
\hline & $\begin{array}{l}\text { Anterior sclera } \\
\text { Mean }(S D)\end{array}$ & $\begin{array}{l}\text { Posterior sclera } \\
\text { Mean }(S D)\end{array}$ \\
\hline Control & $194(65)$ & $121(38)$ \\
7-Methylxanthine & $163(70) \mathrm{p}<0.0001^{\star}$ & $171(48) \mathrm{p}<0.0001^{\star}$ \\
Theobromine & $156(72) \mathrm{p}<0.0001^{\star}$ & $194(53) \mathrm{p}<0.0001^{\star}$ \\
Acetazolamide & $171(58) \mathrm{p}<0.0001^{\star}$ & $94(32) \mathrm{p}<0.0001^{\star}$ \\
L-ornithine & $172(49) \mathrm{p}<0.0009^{\star}$ & $87(30) \mathrm{p}<0.0001^{\star}$ \\
p Value in Kruskal-Wallis H test & $<0.0001$ & $<0.0001$ \\
\hline
\end{tabular}

^Indicates $\mathrm{p}$ values from exact Mann-Whitney test in comparison with controls.

or theobromine $(\mathrm{p}<0.0001)$, and significantly smaller in rabbits treated with acetazolamide or L-ornithine $(\mathrm{p}<0.0001)$ compared with controls. A positive correlation between fibril diameter and contents of hydroxyproline and proline was found in posterior sclera, with a Pearson correlation coefficient 0.870 $(\mathrm{p}=0.055)$ and $0.902(\mathrm{p}=0.037)$, respectively (Table 6).

\section{Discussion}

In rats, a significant growth promoting effect of $0.1 \%$ dietary theobromine has been found, ${ }^{8}$ while a decrease in body weight gain is seen with high doses $(250 \mathrm{mg} / \mathrm{kg}) .{ }^{9}$ Hydroxylysine, hydroxyproline, and to a lesser extent proline are widely used to estimate the content of collagen in connective tissue.

The present results seem to indicate that long term treatment with theobromine or 7-methylxanthine increases the concentration of collagen, and also the thickness of collagen fibrils in posterior sclera from young rabbits. The total concentration of glycosaminoglycans in posterior sclera, as estimated by uronic acid, was reduced by 7-methylxanthine, while there was a near significant increase in the concentration of a specific glycosaminoglycan, dermatan sulphate.

In connective tissue, proteodermatan sulphate is assembled in close proximity to collagen. ${ }^{10}$ An increase in scleral dermatan sulphate can therefore be expected to accompany an increase in collagen. The inverse relation found between content of collagen and uronic acid seems plausible, since thicker and more densely packed collagen fibrils leave less space for glycosaminoglycans.

What is the significance of scleral biochemistry and ultrastructure in relation to the function of the eye?

Dermatan sulphate proteoglycans play a key role in the organisation of collagen, tying fibrils together, restricting their movements in lateral and axial directions, and acting as yardsticks to determine interfibrillar distance. ${ }^{11}$

Collagen is an important component of connective tissue, and determines the biomechanical properties of the tissue. In sclera, it accounts for $80 \%$ of the dry weight, and is responsible for the strength and resilience of the tissue; $90 \%$ is collagen type I, the rest type III. ${ }^{12}$ Changes in the content or organisation of scleral collagen may be of importance in several common disorders of the eye. A preponderance of collagen fibrils with a small diameter is found in myopic sclera, a picture interpreted as "derangement of the growth and organisation of the fibrils". ${ }^{13}$
During development of experimental deprivation myopia in mammals, a decrease of both proteoglycans and collagen is seen in sclera, ${ }^{14}$ and when the deprivation is stopped, a decrease in myopia is seen, accompanied by an increase in the content of proteoglycans. ${ }^{15}$ The changes in scleral biochemistry with deprivation myopia are apparently most pronounced in the posterior part. ${ }^{16}$

Experimentally, monkeys show a significantly smaller mean diameter of collagen in the outer layer of the sclera in myopic eyes compared with controls. ${ }^{17}$

According to one theory, myopia is caused by excessive passive stretching of the scleral tissue. If the development of axial myopia among humans has anything to do with an insufficient concentration of collagen and/or poor quality of collagen fibrils in the sclera, preventive treatment of myopia with 7-methylxanthine, theobromine, or other xanthines may be considered.

Neovascular age related macular degeneration has been hypothesised to be caused by increased rigidity of sclera leading to deterioration of the choroidal vascular supply and ultimately choroidal neovascularisation caused by ischaemia. ${ }^{18}$

Earlier studies by the authors showed regional variation in the human scleral collagen, uronic acid, and different glycosaminoglycans. Sclera from around the papilla was found to be rich in collagen and dermatan sulphate compared with sclera from the equator. ${ }^{79}$ It was also found that scleral content of collagen decreases markedly with age (K Trier, unpublished results). Dermatan sulphate in human sclera decreases with age. ${ }^{20}$

Equatorial sclera is stiffer than posterior, and the rigidity of sclera increases universally with age.$^{21}$ It may thus be assumed that the increasing rigidity of sclera with age is at least partially related to a gradual reduction in the content of collagen. Increased rigidity of sclera has been reported in patients with chronic glaucoma. ${ }^{22} \mathrm{~A}$ dramatic reduction in collagen was found in scleral samples from four patients with absolute glaucoma, most pronounced in the peripapillary region ( $\mathrm{K}$ Trier, unpublished results). These findings are corroborated by a study showing reduced collagen density in peripapillary sclera from human glaucoma eyes. ${ }^{23}$ The production of collagen and elastin in other human tissues, such as skin, decreases with age, and a stimulation of collagen synthesis in elderly people by pharmacological means could conceivably have beneficial effects on skin physiology. ${ }^{24}$ Topical treatment with oestrogens increases the elasticity of aged skin, and increases the number of collagen fibrils in biopsies. ${ }^{25}$

Age related macular degeneration and chronic open angle glaucoma are both common diseases of senescence. It may be worth investigating further whether these conditions could be related to loss of collagen leading to increasing rigidity of sclera, interfering with the blood supply of the choroid and anterior optic nerve. If so, at least theoretically, a treatment that increases or conserves the collagen and/or elastin in sclera would be beneficial for the risk group of elderly people. 
The xanthines examined have no serious side effects, and may thus be applicable in doses comparable with those used in the present study. Based on the results presented, the caffeine and theobromine metabolite 7-methylxanthine may be particularly useful.

The apparent loss of collagen induced by chronic treatment with acetazolamide should be taken into consideration as a potentially harmful side effect. However, the normal clinical dose of acetazolamide is $500 \mathrm{mg}$ per day, equivalent to $8.3 \mathrm{mg}$ per $\mathrm{kg}$ body weight per day for a person weighing $60 \mathrm{~kg}$, which is less than the $30 \mathrm{mg}$ per $\mathrm{kg}$ body weight given in the present study.

The animals used in this study were immature, which means that the effects may not be found in adult animals, since major differences exist between immature and mature sclera-for instance, in the degree of cross linking of the collagen. On the other hand, the young age of the animals may render the results more relevant as regards the development of myopia among children.

As to the mechanism of action of xanthines and acetazolamide on the sclera, no definite conclusion is possible from the present study. It is, however, remarkable that various xanthines, among which caffeine is known to increase the activity of the retinal pigment epithelium, increase the concentration of scleral collagen as well as the thickness of the collagen fibrils, while acetazolamide, known to decrease the activity of retinal pigment epithelium, decreases both the concentration of scleral collagen and the thickness of the collagen fibrils.

A direct effect on the fibroblasts in sclera seems unlikely. In vitro, the methylxanthine pentoxifylline in a very high concentration is known to inhibit the synthesis of extracellular matrix by isolated fibroblasts. ${ }^{26}$ Furthermore, if the effect was a direct one, one would expect a similar effect on anterior and posterior sclera. The results of the present study point at a very different effect of 7-methylxanthine on anterior and posterior sclera, regarding both collagen concentration and fibril diameter.

A theoretically possible explanation is that the fibroblasts in sclera respond to the electrical field generated by the nearby retinal pigment epithelium. This is not totally out of question, since it is known that fibroblasts in culture react to electrical fields of about the same size as that created by the retinal pigment epithelium. ${ }^{27}$ The fibroblasts assume a elongated form, and from other experiments it is known that the shape of the fibroblast determines the synthesis of extracellular material. ${ }^{28}$ Owing to the anatomy of the eye, the eyeball is an electric dipole, with the positive pole at the apex of cornea and the negative pole at the fovea centralis. This could explain the very different effect on anterior and posterior sclera found in this study.

Form deprivation myopia induces activation of scleral matrix metalloproteinase-2 (MMP-2) in the tree shrew. During recovery from deprivation induced myopia, a reduced level of MMP-2 was seen. MMP-2 gene expression is regulated by cytokines and growth factors. Activation of latent MMP-2 may be controlled by the plasminogen-plasmin system. The existence of these or other factors may represent another pathway for the modulation of eye growth by retinal signals. $^{29}$ However, it is difficult to believe that a messenger substance liberated from the retinal pigment epithelium could act locally on the sclera, since such a substance would probably be washed away and diluted by the high blood flow of the choroid.

1 Müller-Limroth W. Die Koffein-Wirkung auf das Elektroretinogram des Menschen. Artzneimittelforschung 1966;20:86-9.

2 Kawasaki K, Mukoh S, Yonemura D, et al. Acetazolamidenduced changes of the membrane potentials of the retina pigment epithelial cell. Doc Ophthalmol 1986;63:375-81.

3 Cox SN, Weinstein G, Arden GB, et al. The effect of acetazolamide on electro-oculogram potential. ARVO Abstracts. Invest Ophthalmol Vis Sci 1988;29:146.

4 Hiroi K, Yamamoto F, Honda Y. Effects of ornithine on the electroretinogram in cat retina. Invest Ophthalmol Vis Sci 1995;36:1732-7.

5 Björnsson S. Simultaneous preparation and quantitation of proteoglycans by precipitation with alcian blue. Anal Biochem 1993;210:282-91.

6 Blumenkrantz N, Asboe-Hansen G. New method for quantitative determination of uronic acids. Anal Biochem 1973;54:484-9.

7 Trier K, Olsen EB, Ammitzbøll T. Regional glycosaminoglycan composition of the human sclera. Acta Ophthalmol 1990;68:304-6.

8 Zoumas BL, Tarka SM. The effect of dietary theobromine on food intake and growth of rats. Fed Proc 1976;35:341.

9 Wang Y, Waller DP, Hikim AP, et al. Reproductive toxicity of theobromine and cocoa extract in male rats. Reprod Toxicol 1992;6:347-53.

10 Young RD. The ultrastructural organization of proteoglycans and collagen in human and rabbit scleral matrix. $\mathcal{f}$ Cell Sci $1985 ; 74: 95-104$

11 Scott JE. Proteoglycan-fibrillar collagen interactions in tissues: dermatan sulphate proteoglycan as a tissue organizer. In: Scott JE, ed. Dermatan sulphate proteoglycans. Portland Press, 1993:165-81.

12 Bailey AJ. Structure, function and agening of the collagens of the eye. Eye $1987 ; 1: 175-83$.

13 Curtin B. The myopias. Basic science and clinical management. Philadelphia: Harper and Row, 1985:255-8.

14 Norton TT, Rada JA. Reduced extracellular matrix in mammalian sclera with induced myopia. Vis Res 1995;35:127181.

15 McBrian NA, Lawlor P. Increased proteoglycan synthesis in the sclera of tree shrew eyes recovering from form deprivation myopia. Invest Ophthalmol Vis Sci 1995;36:S760.

16 Reeder AP, McBrian NA. Biochemical changes in the sclera of tree shrews with a high degree of experimental myopia. Ophthalmic Physiol Opt 1993;13:105.

17 Funata M, Tokoro T. Scleral changes in experimentally myopic monkeys. Graefes Arch Clin Exp Ophthalmol 1990;228:174-9.

18 Friedman E, Ivry $\mathrm{M}$, Ebert $\mathrm{E}$, et al. Increased scleral rigidity and age-related macular degeneration. Ophthalmology 989:96:104-8

19 Trier K, Olsen EB, Ammitzbøll T. Collagen and uronic acid distribution in the human sclera. Acta Ophthalmol 1991;69: $99-101$

20 Brown CT, Vural M, Johnson M, et al. Age-related changes of scleral hydration and sulfated glycosaminoglycans. Mech Ageing Dev 1994;77:97-107.

21 Friberg TR, Lace JW. A comparison of the elastic properties of human choroid and sclera. Exp Eye Res 1988;47:429-36. 2 Friedenwald JS. Contribution to the theory and practice of tonometry. Am f Ophthalmol 1937;20:985.

23 Quigley HA, Dorman Pease ME, Brown AE. Quantitative study of collagen and elastin of the optic nerve head and sclera in human and experimental monkey glaucoma. Curr Eye Res 1991;10:877-88.

24 Uitto J, Fazio MJ, Olsen DR. Molecular mechanisms of cutaneous aging. F Am Acad Dermatol 1989;21:614-22

25 Schmidt JB, Binder M, Demschik G, et al. Treatment of skin aging with topical estrogens. Int F Dermatol 1996;35:66974

26 Windmeier C, Gressner AM. Pharmacological aspects of pentoxifylline with emphasis on its inhibitory actions on hepatic fibrogenesis. Gen Pharmacol 1997;29:181-96.

27 Erickson CA, Nuccitelli R. Embryonic fibroblast motility and orientation can be influenced by physiological electric fields. F Cell Biol 1984;98:296-307.

28 Evangilisti R, Becchetti E, Locci P, et al. Coordinate effects of concanavalin A on cytoskeletal organization, cell shape, lycominoglycan a cymution and ity in chick embryonic cultured fibroblasts. Eur $\mathcal{F}$ Histochem 1993;37:161-72.

29 Guggenheim JA, McBrian NA. Form-deprivation myopia induces activation of scleral matrix metalloproteinase-2 in tree-shrew. Invest Ophthalmol Vis Sci 1996;37:1380-95. 